\title{
Vitamin D status among pulmonary tuberculosis patients and controls in Tanzania
}

\author{
H. Friis ${ }^{1}$, N. Range ${ }^{2}$, J. Changalucha ${ }^{3}$, G. PrayGod ${ }^{3}$, K. Jeremiah ${ }^{3}$, D. Faurholt-Jepsen ${ }^{1}$, H. Krarup ${ }^{4}$, \\ C. Mølgaard ${ }^{1}$ and A. B. Andersen ${ }^{5}$ \\ ${ }^{1}$ Department of Human Nutrition, University of Copenhagen, Denmark, ${ }^{2}$ Muhimbili Medical Research Centre, NIMR, \\ Dar es Salaam, Tanzania, ${ }^{3}$ Mwanza Medical Research Centre, NIMR, Mwanza, Tanzania, ${ }^{4}$ Department of Clinical \\ Biochemistry, Aalborg University Hospital, Aalborg, Denmark and ${ }^{5}$ Department of Infectious Diseases, Odense University \\ Hospital, Odense, Denmark
}

Vitamin D may be a determinant of tuberculosis $(\mathrm{TB})^{(1)}$, yet the evidence is inconclusive. To assess the role of pulmonary TB (PTB), HIV and acute phase response as predictors of serum $25(\mathrm{OH}) \mathrm{D}$, we conducted a sex- and age-matched cross-sectional study among PTB patients and non-TB controls. The PTB patients were categorised as sputum negative (PTB - ) and positive $(\mathrm{PTB}+)$ based on culture. For 355 cases, an age- and sex-matched non-TB neighbourhood control was randomly selected. HIV status and serum S-25(OH)D, CRP and AGP were determined. Linear regression analysis was used to assess predictors of S-25(OH)D.

Vitamin D data were available on $97.8 \%$ of 1605 participants. Mean (sD) S-25(OH)D was 84.4 (25.6) nmol/1 with 39.6\% below $75 \mathrm{nmol} / \mathrm{l}$ among 346 controls, and 110.9 (35.7) nmol/l and 15.0\% among $1223 \mathrm{PTB}$ patients. Time of recruitment, religion, marital status, occupation, PTB and HIV, and elevated S-AGP were predictors of S-25(OH)D, while age, sex, smoking and alcohol intake were not. S-25(OH)D was highest in 2006 compared to subsequent years, and in the first compared to subsequent quarters. PTB patients had $15.4(95 \%$ CI $11.2,19.7) \mathrm{nmol} / \mathrm{l}$ higher S-25(OH)D than controls, and HIV + had $9.1(95 \%$ CI 5.5, 12.7) nmol/l higher levels than HIV patients. As seen in the Table, elevated S-AGP was a positive predictor of S-25(OH)D, and explained most of the difference by PTB, but not HIV, status.

\begin{tabular}{|c|c|c|c|c|c|c|}
\hline & \multicolumn{3}{|c|}{ Model 1} & \multicolumn{3}{|c|}{ Model 2} \\
\hline & B & $95 \% \mathrm{CI}$ & $\mathrm{P}$ & B & $95 \% \mathrm{CI}$ & $\mathrm{P}$ \\
\hline \multicolumn{7}{|l|}{ PTB status } \\
\hline Non-TB control & - & & & & & \\
\hline PTB - & 15.9 & $10.7,21,0$ & $<0.001$ & 7.6 & $1.1,14.1$ & 0.02 \\
\hline PTB + & 15.3 & $10.9,19.7$ & $<0.001$ & 4.6 & $-2.4,11.6$ & 0.19 \\
\hline \multicolumn{7}{|l|}{ HIV status } \\
\hline HIV - & - & & & - & & \\
\hline $\mathrm{HIV}+$ & 9.1 & $5.5,12.7$ & $<0.001$ & 7.9 & $4.3,11.6$ & $<0.001$ \\
\hline \multicolumn{7}{|l|}{ Serum AGP (mg/l) } \\
\hline$<1$ & & & & - & & \\
\hline $1-2$ & & & & 11.9 & $5.6,18.2$ & $<0.001$ \\
\hline $2-3$ & & & & 12.5 & $5.7,19.2$ & $<0.001$ \\
\hline $3+$ & & & & 14.0 & $6.5,21.4$ & $<0.001$ \\
\hline
\end{tabular}

In conclusion, hypovitaminosis D was common in Tanzania, with considerable secular and seasonal variation. In contrast to previous studies $^{(2)}$, PTB and HIV and elevated acute phase response were associated with higher S-25(OH)D. This could be because infections increasing S-25(OH)D, either due increased vitamin D status or to an effect on the validity of S-25(OH)D as a marker of vitamin D status during the acute phase response.

Supported by Danish Council for Independent Research (grant 22-04-0404), by Danida (104.Dan.8-898), and the University of Copenhagen (Cluster in International Health)

1. Nnoaham KE \& Clarke A (2008) Low serum vitamin D levels and tuberculosis: a systematic review and meta-analyses. Int J Epidemiology 37, $113-119$.

2. Friis H, Range N, Pedersen ML et al. (2008) Hypovitaminosis D is common among pulmonary tuberculosis patients in Tanzania but is not explained by the acute phase response. $J$ Nutr 138, 2474-2480. 